LHC Project Note 411

2008-02-26

Christine.hoa@cern.ch, mokhov@fnal.gov

\title{
Inter-comparison of MARS and FLUKA: Predictions on Energy Deposition in LHC IR Quadrupoles
}

\author{
C. Hoa / CERN, AT-MCS-MA, N.V Mokhov / Fermilab Accelerator Physics Center, \\ F. Cerutti, A. Ferrari / CERN, AB-ATB-EET / CERN
}

Keywords: energy deposition, magnets

\begin{abstract}
Summary
Detailed modellings of the LHC insertion regions (IR) have earlier been performed to evaluate energy deposition in the IR superconducting magnets [1-4]. Proton-proton collisions at $14 \mathrm{TeV}$ in the centre of mass lead to debris, depositing energy in the IR components. To evaluate uncertainties in those simulations and gain further confidence in the tools and approaches used, inter-comparison calculations have been performed with the latest versions of the FLUKA (2006.3b) [5, 6] and MARS15 [7, 8] Monte Carlo codes. These two codes, used worldwide for multi particle interaction and transport in accelerator, detector and shielding components, have been thoroughly benchmarked by the code authors and the user community (see, for example, recent $[9,10]$ ). In the study described below, a better than $5 \%$ agreement was obtained for energy deposition calculated with these two codes - based on different independent physics models - for the identical geometry and initial conditions of a simple model representing the IR5 and its first quadrupole.
\end{abstract}

\section{Introduction}

In order to compare energy deposition results with FLUKA 2006.3 [5, 6] and MARS 15 $[7,8]$, the settings of the simulation models have to be exactly the same so the different results can only come from the physics of the codes. In detailed models of the nominal LHC insertion regions [1-4], the geometry layout and the magnetic field definitions are based on many input parameters. They can differ depending on the different sources available through the period over which the works have been carried out. Hence, an inter-comparison work applied on a simplified model with very few components is a relevant exercise.

\section{IR5 toy model}

\section{$2.1 \quad$ Geometry layout}

The simplified geometry, representing the main features of the inner triplet quadrupole and IR5 layout, has cylindrical symmetry. It includes the copper collimator 'Target Absorber Secondaries (TAS)', the first quadrupole magnet Q1 and the stainless steel beam pipe in the magnet (Figure 1). The Q1 magnet (MQXA) has 4 layers of superconducting (SC) cables, an iron yoke, super-insulation and a stainless steel cryostat vessel (Figure 2). The inner cable, 
cable 1, is the closest to the beam pipe and is the most interesting as it is the region where the maximum energy deposition in SC occurs. The figures are generated with FLAIR [11], FLUKA advanced interface.

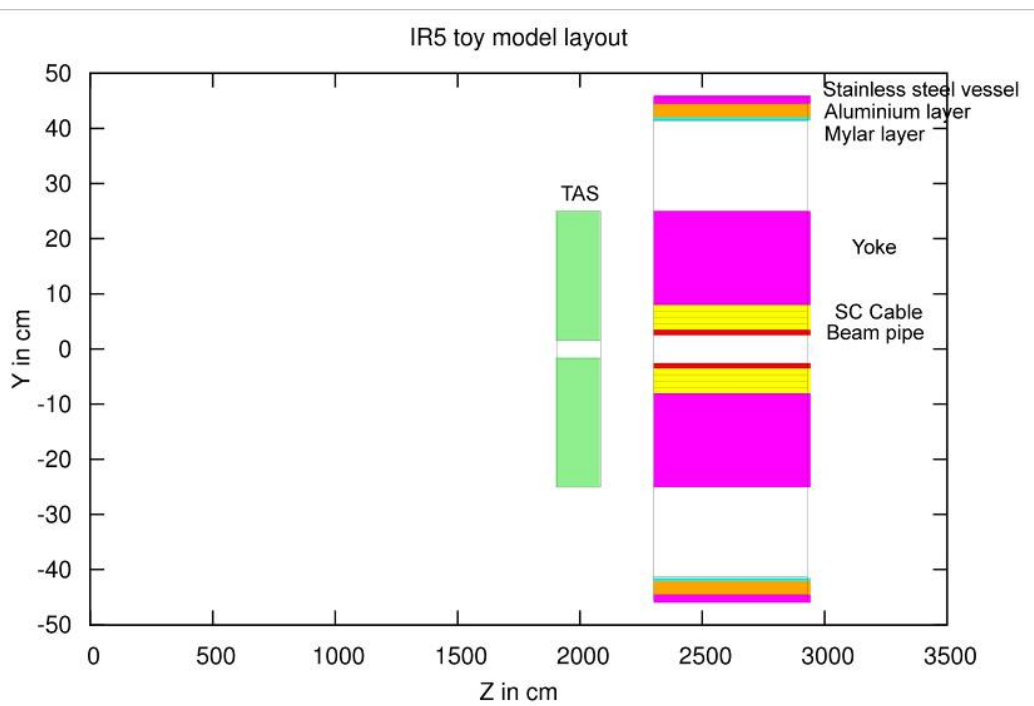

Figure 1: Geometry layout with FLUKA

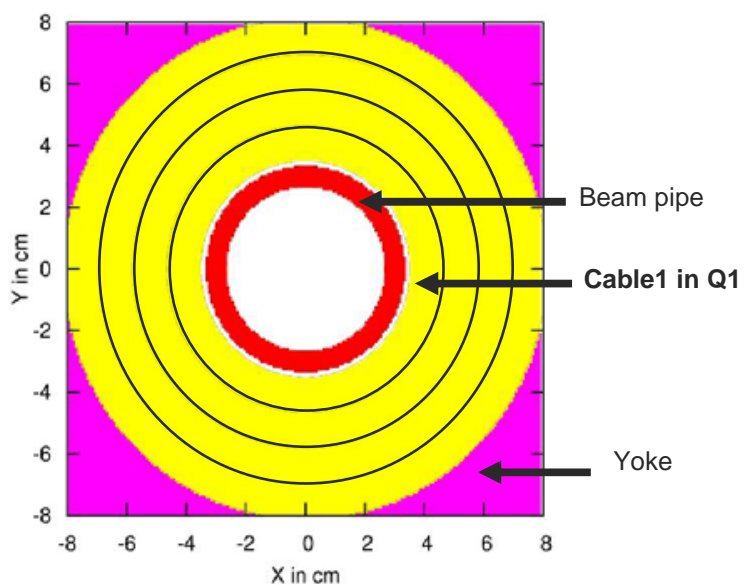

Figure 2: Q1 cross section

\section{$2.2 \quad$ Magnetic field description}

The detector magnetic field description has been simplified with a constant solenoidal field of $\mathrm{Bz}=4$ Tesla, up to $6.5 \mathrm{~m}$ from the interaction point (IP) (Figure 3). A bi-dimensional map of the quadrupole magnetic field for Q1 has been implemented (Figure 4). It is a MQXA magnet with an operating gradient of $199.4591 \mathrm{~T} / \mathrm{m}$. For the interaction debris, the magnet is focusing in the horizontal plane for a positive charged particle (Figure 5). 


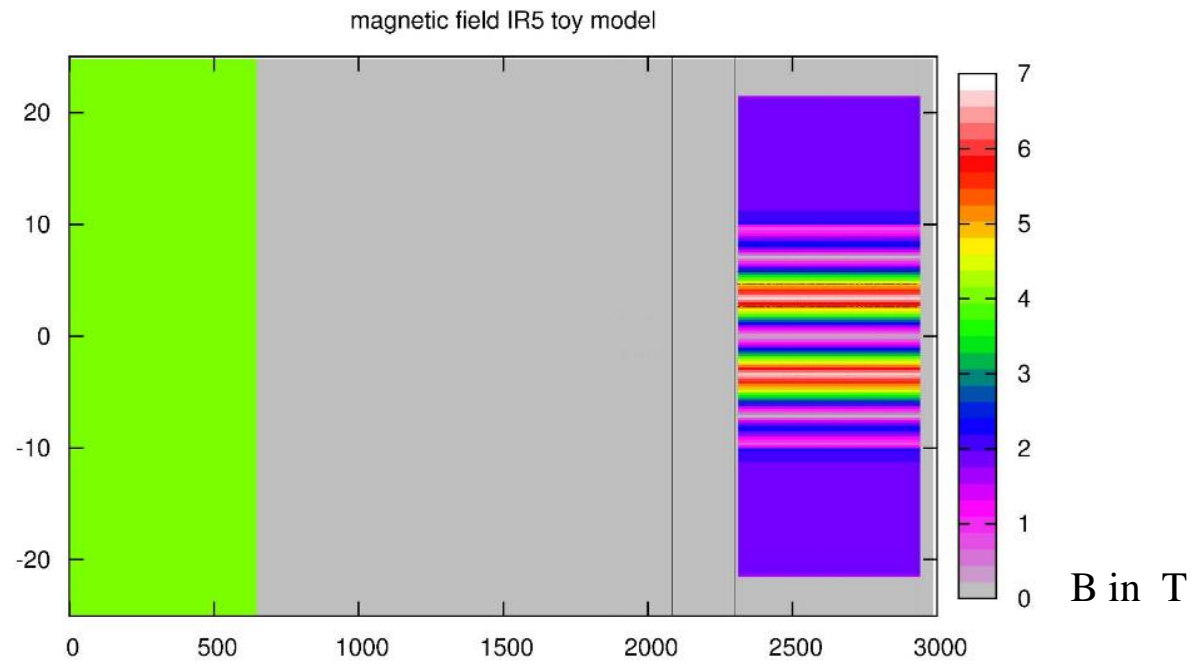

Figure 3: Longitudinal view of the magnetic field in the IR5 toy model
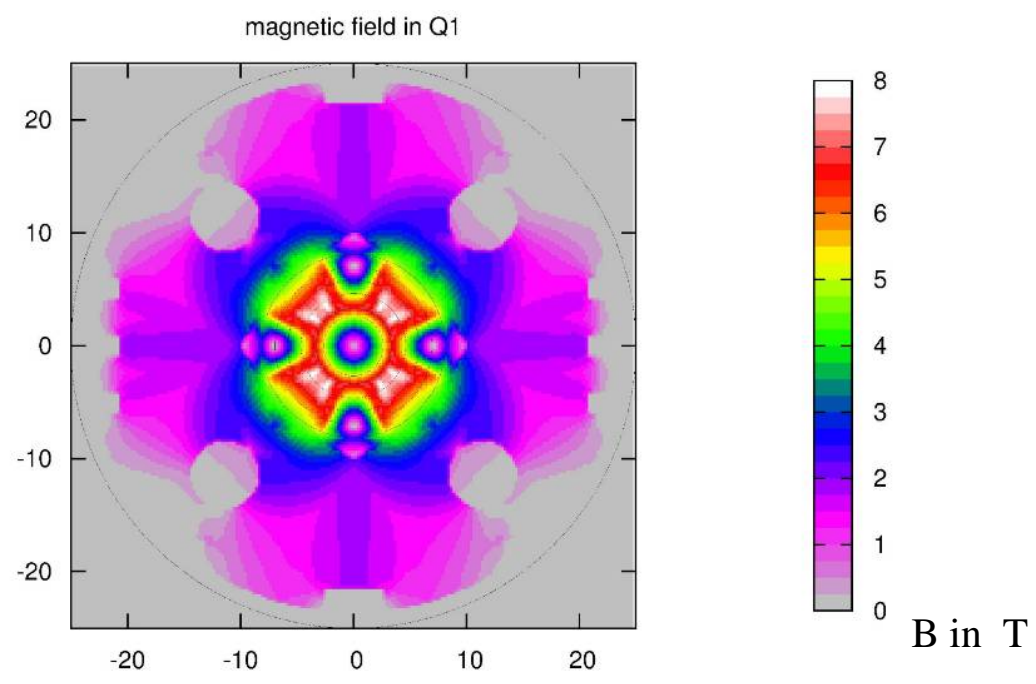

Figure 4: Q1 cross section showing the magnetic field intensity

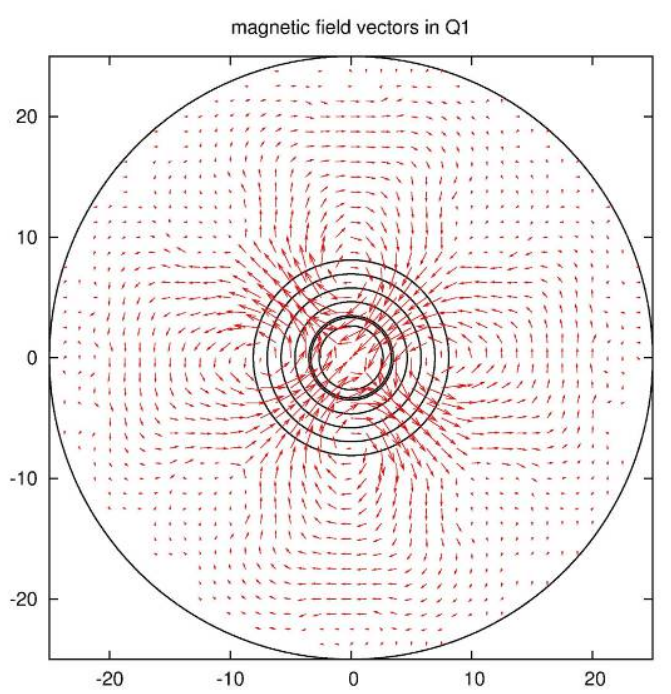

Figure 5: Q1 cross section showing the magnetic map

\section{$2.3 \quad$ Particle tracking}

Both FLUKA and MARS have coordinates defined with a right handed system, but the definition of the axes is not the same (Figure 6).
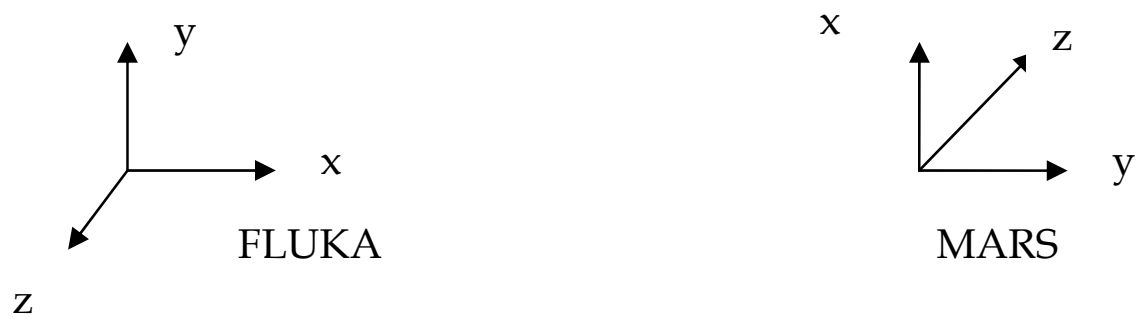

Figure 6: Right handed coordinate systems. The $z$ axis coincides with the beam 1 axis, i.e the direction of the protons beam, coming out from the IP. With FLUKA, the x-y plane is seen from the end of the insertion, whereas with MARS, the $y-x$ plane is seen from the IP. 
The event-generator used to simulate the proton-proton collisions at the center of mass energy of $14 \mathrm{TeV}$ is DPMJET III [12] for the 2 codes. A half horizontal crossing angle of $142.5 \mu \mathrm{rad}$ is modelled in the IR5 [13].

Figure 7, generated with MARS15 Graphical User Interface (GUI), shows some secondary particles tracked in the insertion region. Each colour corresponds to one type of secondary particle. The neutrons have been deleted as they are too numerous in the TAS and would have hidden the components.

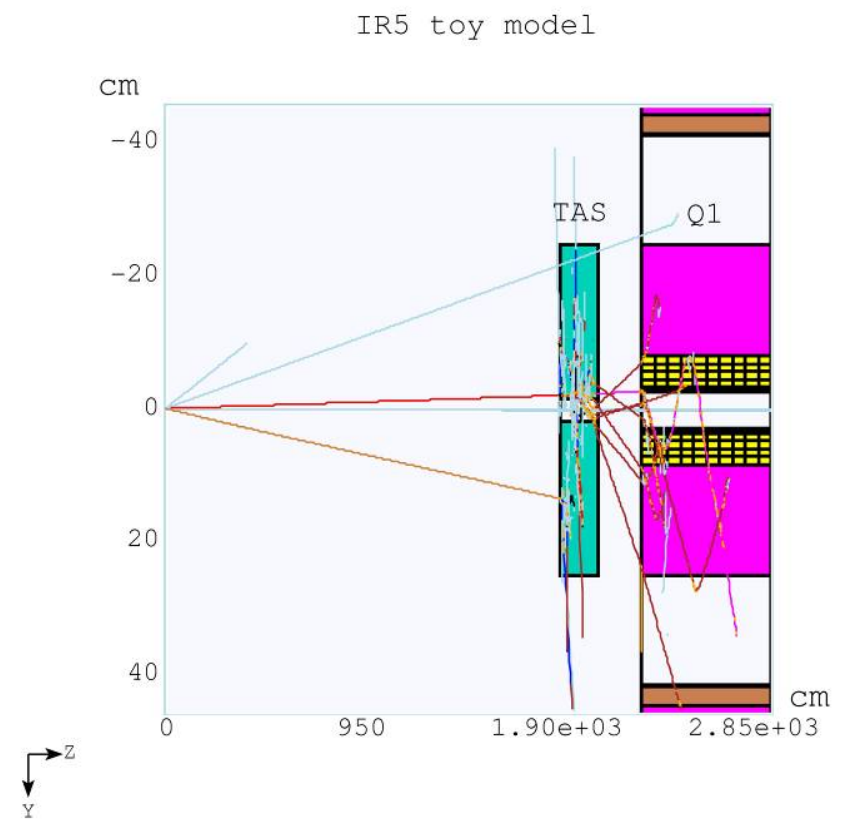

Figure 7: Secondary particles traced in the IR5 toy model (MARS15)

\section{$2.4 \quad$ Scoring parameters}

For comparing the results of the codes, the scorings have been specified the same way with a Cartesian binning for the front face of the TAS, in the middle plane and in the end plane of the magnet Q1 (Table 1). The XYZ coordinates relate to the FLUKA framework system. A cylindrical mesh split the inner cable of the magnet Q1 in the azimuthal and longitudinal directions (Table 2). The R- $\phi$-Z coordinates relate to the FLUKA framework system.

\begin{tabular}{|l|l|l|l|l|l|l|}
\hline Scoring & $\mathrm{X}$ & $\mathrm{Y}$ & $\mathrm{Z}$ & $\mathrm{NX}$ & $\mathrm{Ny}$ & $\mathrm{Nz}$ \\
\hline TAS & {$[-25: 25]$} & {$[-25: 25]$} & {$[1905: 1925]$} & 150 & 150 & 1 \\
\hline Q1 middle & {$[-25: 25]$} & {$[-25: 25]$} & {$[2600: 2630]$} & 150 & 150 & 1 \\
\hline Q1 End & {$[-25: 25]$} & {$[-25: 25]$} & {$[2900: 2930]$} & 150 & 150 & 1 \\
\hline
\end{tabular}

Table 1: Cartesian Binning

\begin{tabular}{|l|l|l|l|l|l|l|}
\hline Scoring & $R$ & $\phi$ & $Z$ & $N R$ & $N \phi$ & $N z$ \\
\hline Q1 inner cable & {$[3.5: 4.65]$} & {$[0: 360]$} & {$[2300: 2930]$} & 1 & 180 & 63 \\
\hline
\end{tabular}




\section{$2.5 \quad$ Cut off parameters}

The cut off parameters have been chosen to be the same in both FLUKA and MARS (Table 3). The threshold is $0.2 \mathrm{MeV}$ for all the particles except for the neutrons which are followed down to the thermal energy. A difference in the electron and positron cut off energies has been found after this study and write-up were complete: in FLUKA, for the electrons and positrons this value has been increased to $1 \mathrm{MeV}$. For this energy, e+ and e- have a range in copper of less than $1 \mathrm{~mm}$.

\begin{tabular}{|l|l|}
\hline Number of histories & $15000 \mathrm{pp}$-collisions \\
\hline Cut off energy for photons & $200 \mathrm{keV}$ \\
\hline Cut off energy for e-/+e- & $200 \mathrm{keV}$ (MARS), 1 MeV (FLUKA) \\
\hline Cut off energy for Muons & $200 \mathrm{keV}$ \\
\hline Cut off energy for Hadrons & $200 \mathrm{keV}$ \\
\hline Cut off for Neutrons & down to thermal energy \\
\hline
\end{tabular}

Table 3: Cut-off parameters

\subsection{Biasing Parameters}

In both simulations, biasing schemes have been utilized to optimize the CPU time. Leading Particle Biasing (LPB) schemes have been applied for electromagnetic and hadronic interactions. LPB discards a fraction of the secondary particles in hadronic and electromagnetic cascades, provided that the selected ones have properly adjusted weights. This way, the geometrical increase with energy of the number of particles in the cascades is avoided.

The biasing parameters have been chosen independently for each code. The results shall not be influenced by the biasing parameters. The CPU time can differ for MARS and FLUKA due to the biasing schemes but also due to the performance of the computing processors.

\section{Energy deposition results}

\subsection{Normalization factor}

Both codes give the energy deposition in GeV/primary, in our case per proton-proton collision. The following formula (1) gives the transformation to power deposition [Watts], as a function of the luminosity $L$ and the reaction cross section A (including inelastic scattering and single diffraction events).

$$
\operatorname{Power}(W)=\operatorname{Energy}(G e V / \text { collision }) * 10^{9} * 1.602 * 10^{-19} * \text { L }\left(\text { collision. } \mathrm{cm}^{-2} \mathrm{~s}^{-1}\right) * A(\text { barn }) * 10^{-24}
$$

Equation (1), considering the upgrade LHC luminosity, $L=10^{35} \mathrm{~cm}^{-2} \mathrm{~s}^{-1}$ and assuming $\mathrm{A}=$ 80 mbarn, becomes:

$$
\operatorname{Power}(W)=1.28 * \text { Energy }(\mathrm{GeV} / \text { collision })
$$


MARS gives the power deposition density results in $\mathrm{GeV} / \mathrm{g} / \mathrm{collision}$. The conversion into $\mathrm{GeV} / \mathrm{cm}^{3} /$ collision is made with the multiplication by the superconducting cable density assumed to be $7 \mathrm{~g} / \mathrm{cm}^{3}$.

\subsection{Total heat loads}

The Table 4 presents the results of the total heat loads in the different components of the insertion region. The agreement is quite good between the two independent codes FLUKA and MARS, within less than 5\% for most elements. In the most interesting region, the SC coil, the agreement is amazing, within $1 \%$. The yoke region shows a discrepancy of $23 \%$, which can be attributed to differences in the event generators at intermediate energies below a few $\mathrm{GeV}$, cross-section libraries for low-energy neutrons. All of the above are important for welldeveloped cascades in the iron region far from the shower core. Several cross checks have been done and confirmed this difference.

Total heat loads in the insertion region elements $(W)$ for upgrade luminosity $L=10 *$ 0

\begin{tabular}{|l|r|r|r|r|r|}
\hline & FLUKA & + l- (\%) & MARS & +l- (\%) & $\begin{array}{l}\text { Ratio } \\
\text { FLUKA/MARS }\end{array}$ \\
\hline TAS & 1853.7 & 0.5 & 1827.3 & 0.1 & 1.01 \\
\hline Beam pipe & 89.1 & 1.0 & 97.9 & 0.4 & 0.91 \\
\hline Q1 cable & 158.0 & 0.6 & 159.1 & 0.2 & 0.99 \\
\hline yoke & 96.3 & 0.9 & 78.5 & 0.4 & 1.23 \\
\hline aluminium layer & 2.3 & 0.6 & 2.4 & 0.5 & 0.98 \\
\hline mylar insulation & 19.5 & 0.8 & 20.4 & 0.3 & 0.96 \\
\hline stainless steel vessel & 16.8 & 0.8 & 17.3 & 0.3 & 0.97 \\
\hline
\end{tabular}

Table 4: Comparison of total heat loads

\subsection{Power deposition maps}

\subsubsection{Peak power density at the end of Q1, in the inner layer}

To estimate the peak power density in the inner cable (cable1), the binning size for the cross section is based on the cable width $(1.3 \mathrm{~cm} * 1.3 \mathrm{~cm})$. The length of the bin is $30 \mathrm{~cm}$ to have an isothermal bin in the $\mathrm{z}$ direction. The bin volume is around $50 \mathrm{~cm}^{3}$.

In the MARS simulation, the peak value at $\mathrm{z}=2900 \mathrm{~cm}$ averaged over this bin volume is

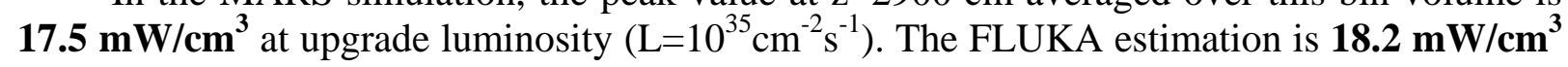
(with $4.4 \%$ statistical error).

\subsubsection{Longitudinal distribution of the power density in the inner layer, averaged over the azimuthal direction.}

There is also a very good agreement for the power density in the inner cable (cable1) along the magnet (Figure 8). The two longitudinal distributions show the same behaviour, with a discrepancy, within $15 \%$, appearing at the end of the magnet. 


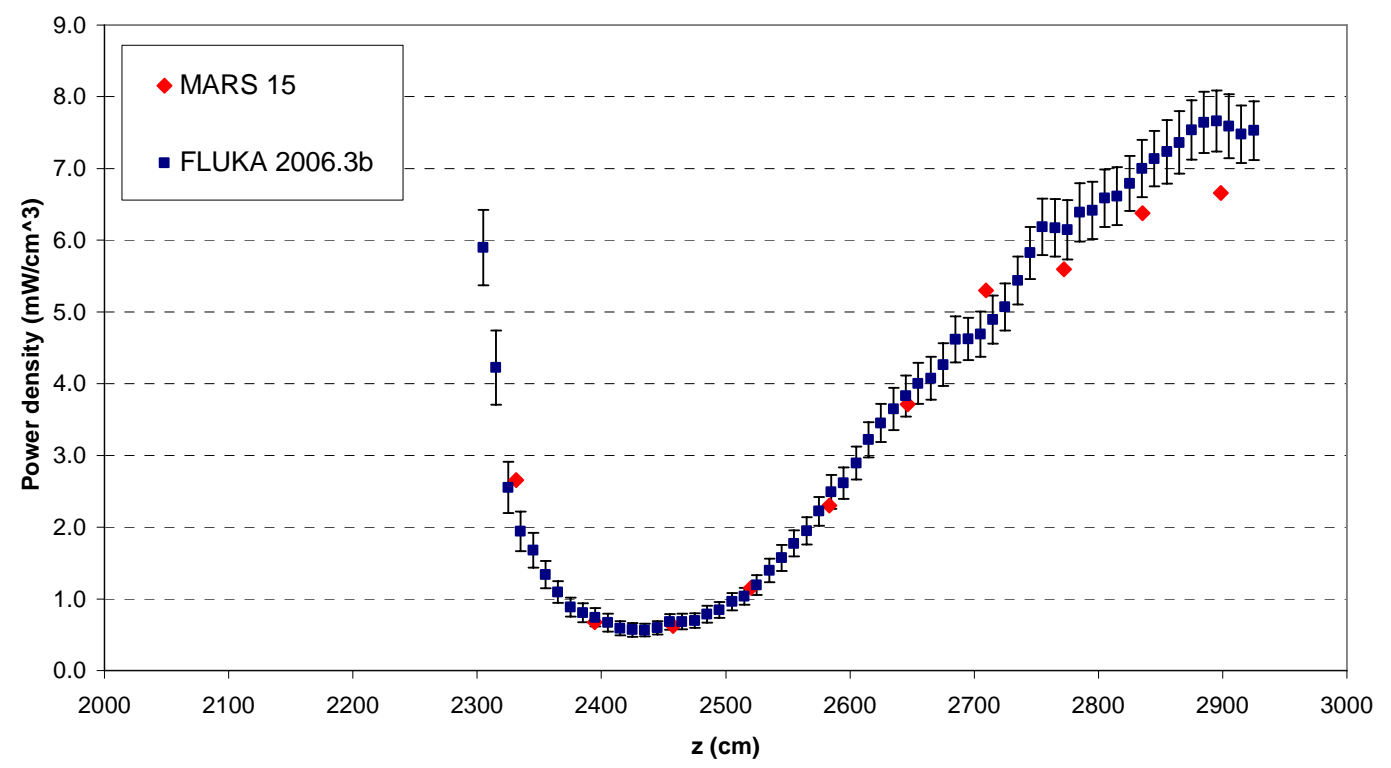

Figure 8: Azimuthal averaged power density in cable1 along Q1

\subsubsection{Lateral profile at the end of Q1}

Figure 9 and Figure 10 show the power density in the Q1 cross section averaged in the $\mathrm{z}$ direction between $z=2900$ and $z=2930 \mathrm{~cm}$ (at the end of the magnet) where the maximum energy deposition occurs. The symmetries in the vertical and horizontal planes are observed on the two plots, showing the good agreement between the 2 codes. The binning is finer in the plot for MARS (Figure 10).

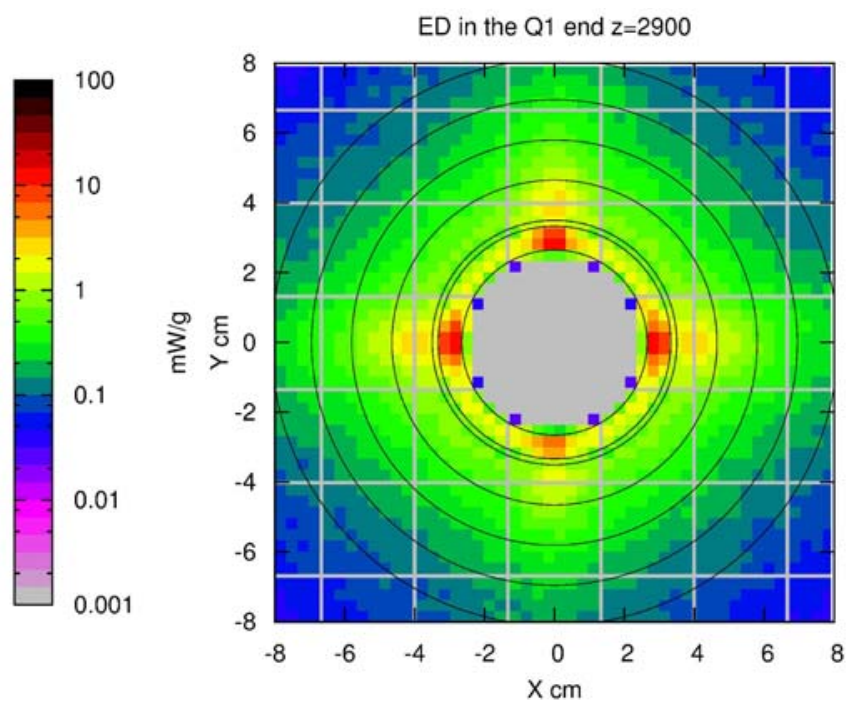

Figure 9: FLUKA, Power deposition map in Q1 averaged over $2900<\mathrm{z}<2930 \mathrm{~cm}$

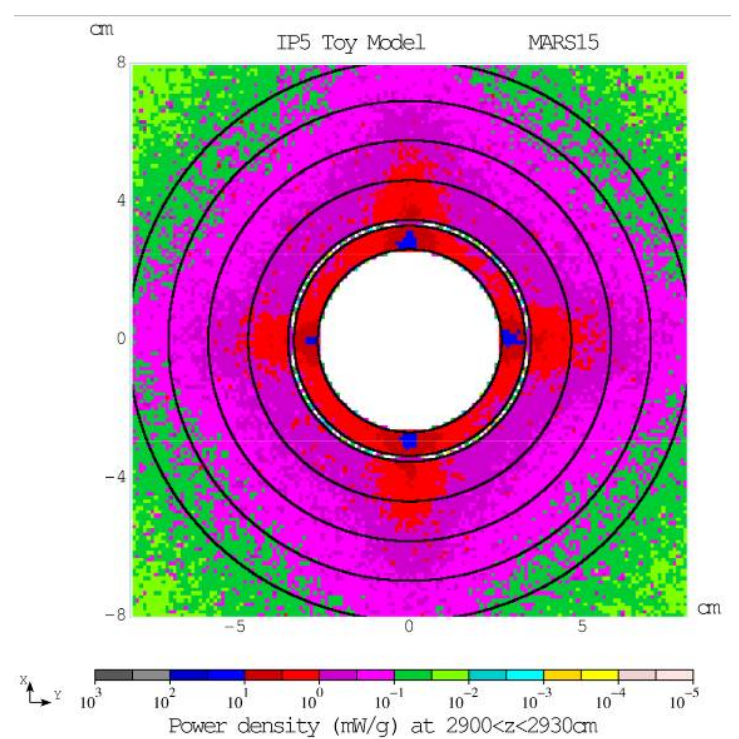

Figure 10: MARS, Power deposition map in

Q1 averaged over $2900<\mathrm{z}<2930 \mathrm{~cm}$ 


\section{Particle spectra in inner cable (cable1)}

The highest power deposition in the magnet occurs in the inner cable $(3.5<\mathrm{r}<4.65 \mathrm{~cm}$, $2900<\mathrm{z}<2930 \mathrm{~cm})$. There, the energy spectra of different types of particles have been investigated.

\subsection{Hadrons}

There is a fairly good agreement between the two codes for the hadron spectra. Minor discrepancies can be observed:

- for protons with $\mathrm{E}>1 \mathrm{GeV}$ (Figure 11),

- for pions and kaons at low energies ( $<100 \mathrm{MeV}$ ), (Figure 12) and

- for neutrons at all energies (Figure 13).

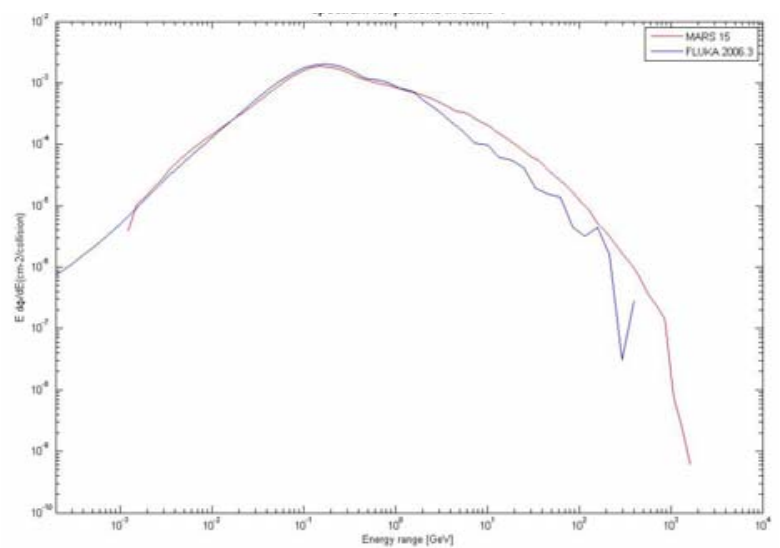

Figure 11 : Proton spectra in cable1

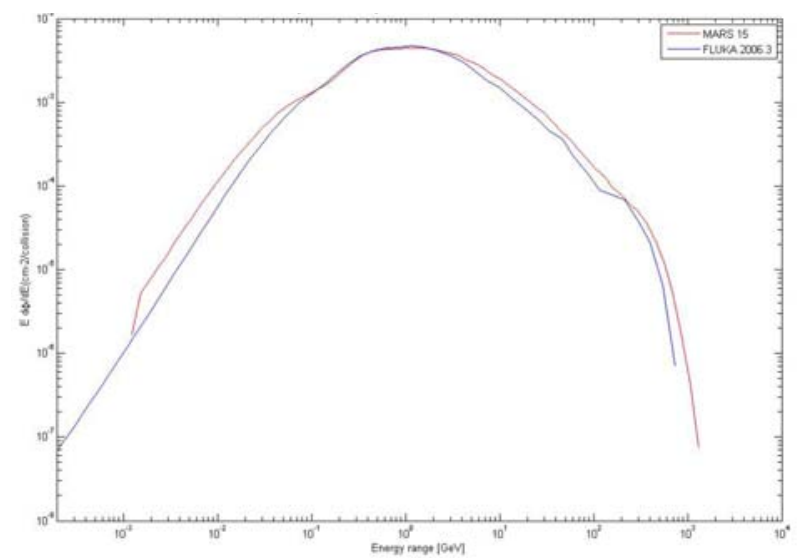

Figure 12: Pion and kaon spectra in cable1

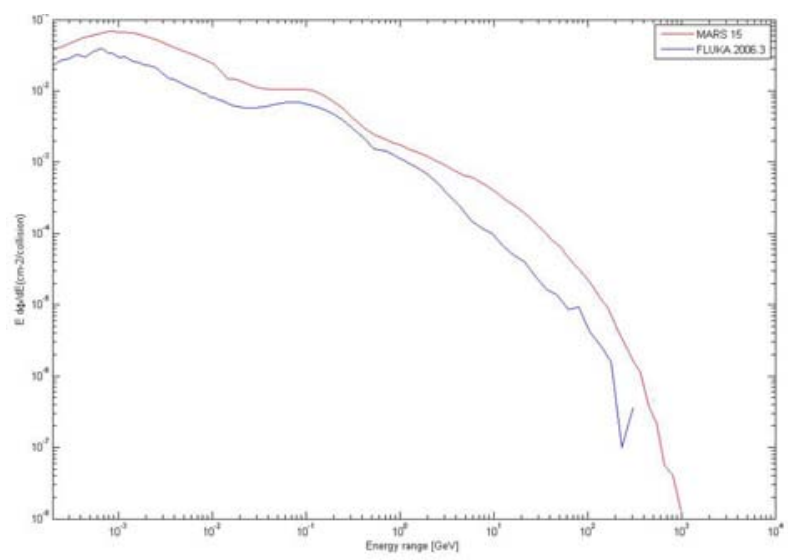

Figure 13: Neutron spectra in cable1 


\subsection{Leptons}

FLUKA and MARS agree almost perfectly well for the electrons and positrons below about $20 \mathrm{GeV}$.

The muon contribution to the total particle fluence and energy deposition in the inner cable is negligible, but it is interesting to compare the results from FLUKA and MARS. Both codes show the same shape for the muon energy spectrum: the first peak corresponds to the products of stopped pion decays, the second peak corresponds to the products of stopped kaon decays, and the remaining higher energy muons are those generated in decays in flight (Fig. 15).

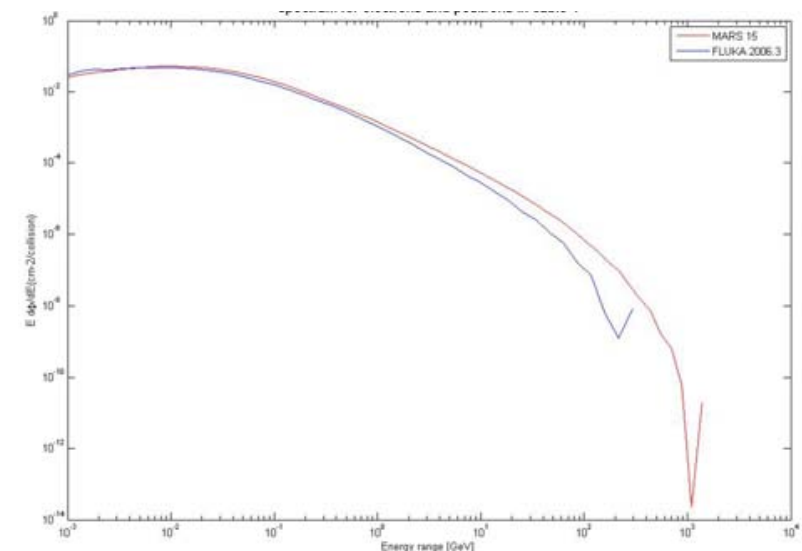

Figure 14: e+ and e- spectra in the inner cable

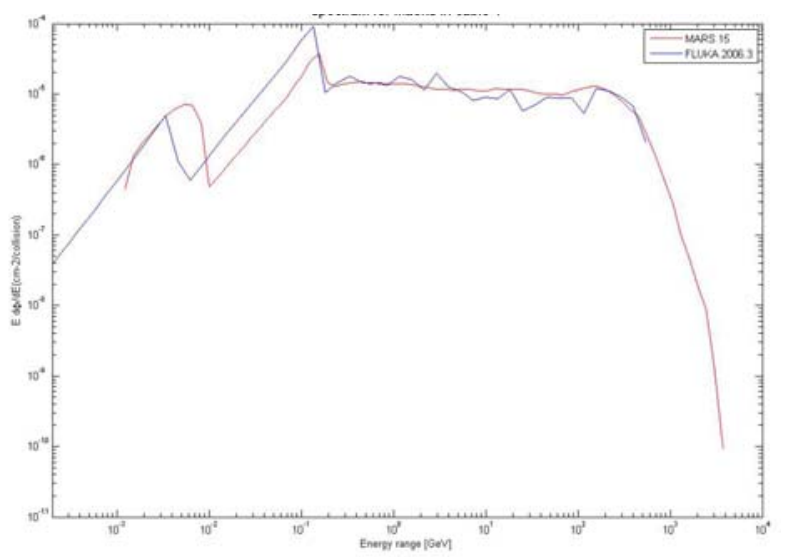

Figure 15: Muon spectra in the inner cable

\subsection{Photons}

The photon spectra are in a good agreement in the low and medium energy ranges. The discrepancy at E> $50 \mathrm{GeV}$ concerns a very small photon flux with larger statistical errors (Figure 16). We attribute the difference in that region to the differences in the neutral pion production models in the codes.

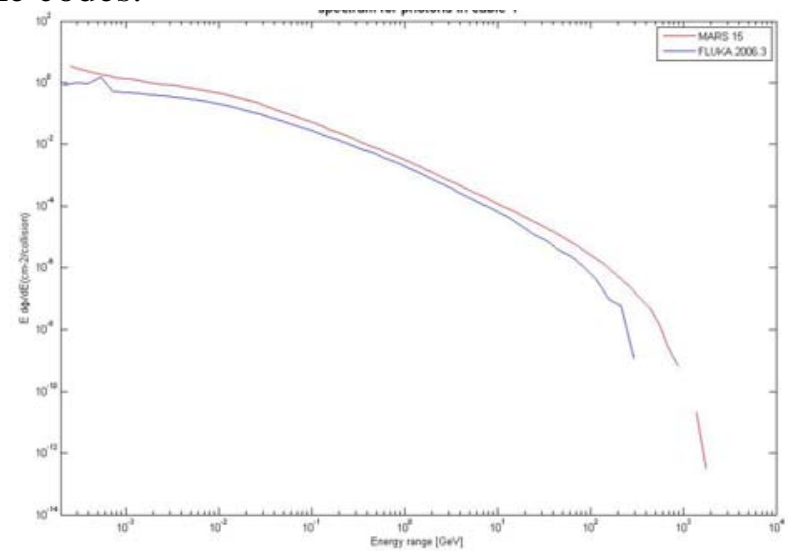

Figure 16: Photon spectra in the inner cable 


\section{Muons: further investigations}

After the detailed analysis of the spectra of the particles in the cable1, the comparison of the muon fluences encourages to carry on further investigations. A simple model of a $2 \mathrm{GeV}$ positive pion beam impacting a carbon and then an iron target was built in order to better compare the physics models for the production and transport of muons, resulting from pion decay (Figure 17).

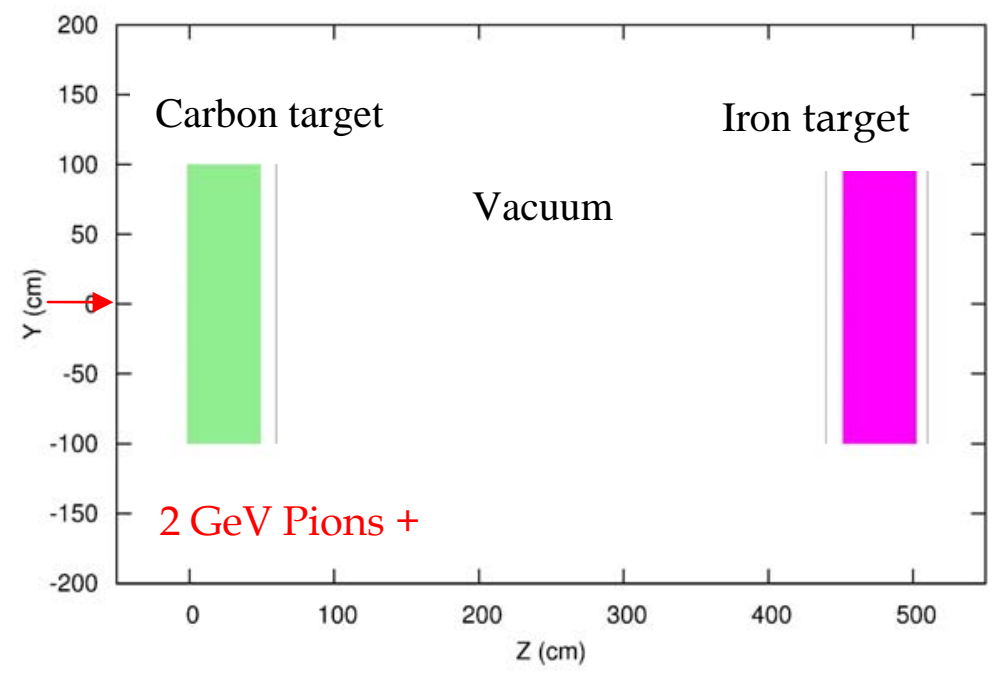

Figure 17: Pion source with targets

Figure 18 presents the longitudinal distribution of the muon fluence. The two distributions are in a good agreement. A small discrepancy between the two curves can be explained by different hadron production models in FLUKA and MARS. It seems that FLUKA generates slightly softer spectra of pions in pion $^{+}$-carbon nuclear interactions at $2 \mathrm{GeV}$. Therefore, FLUKA's secondary pions have somewhat larger angles than those from MARS, and muons from decay of those softer secondary pions have even larger angles compared to the MARS's ones. As a result, we have a higher radial leak for muons in the drift from carbon to iron in the FLUKA case that is reflected in the plot. The spectra "before the iron target" (Figure 19) and the longitudinal fluence distribution are fully consistent.

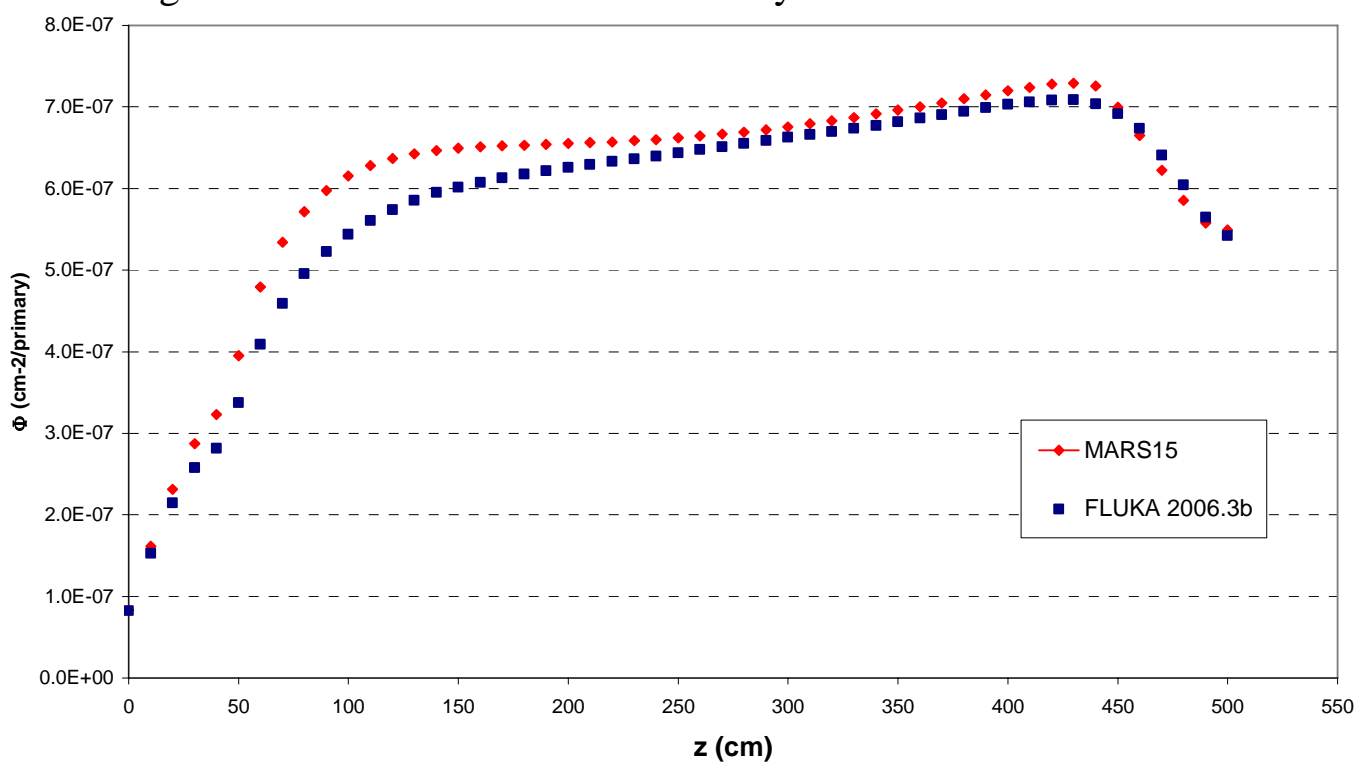

Figure 18: Longitudinal distribution of the muon fluence 
The spectra "after the iron target" (Figure 20) indicates different muon fluences in the medium and high energy ranges $(\mathrm{E}>10 \mathrm{MeV})$, resulting from pion ${ }^{+}$-iron nuclear interactions.

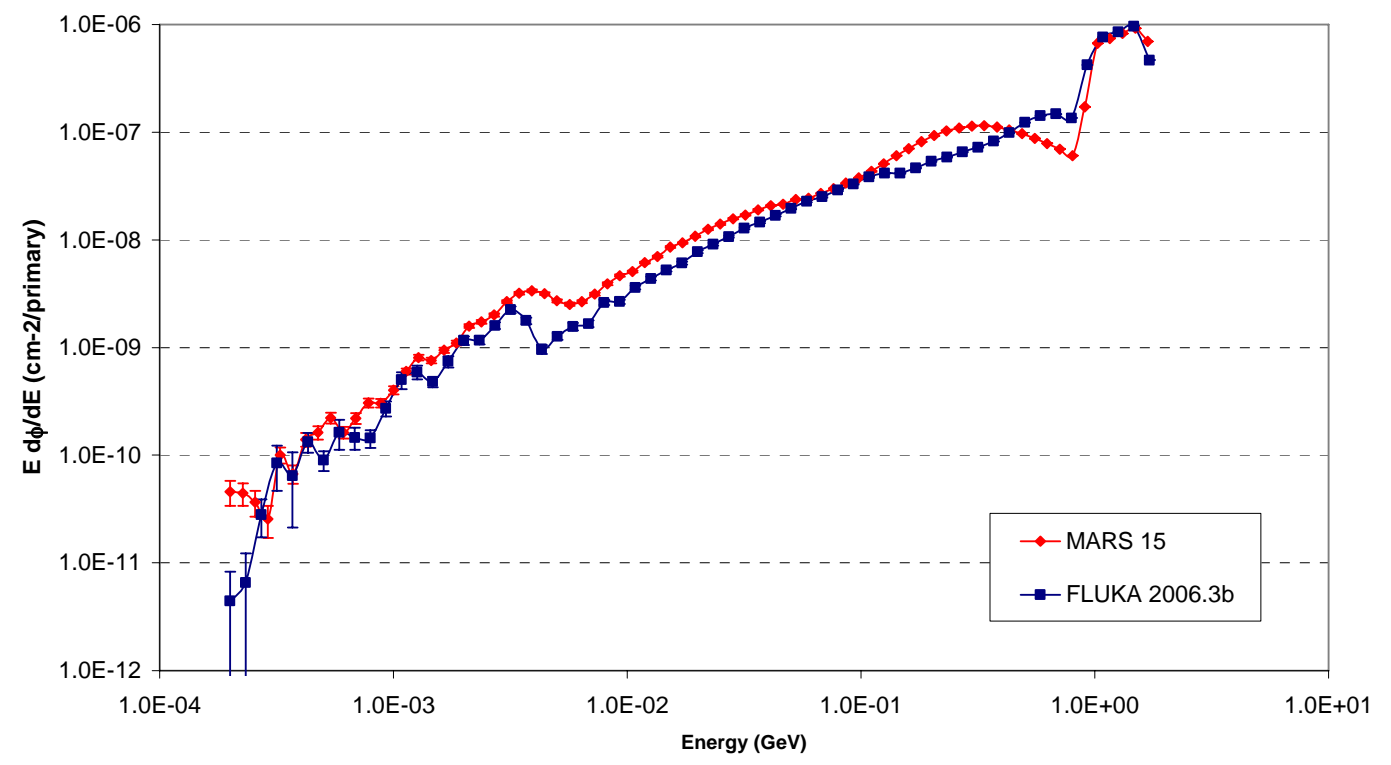

Figure 19: Energy spectra of the muons Before the Iron Target

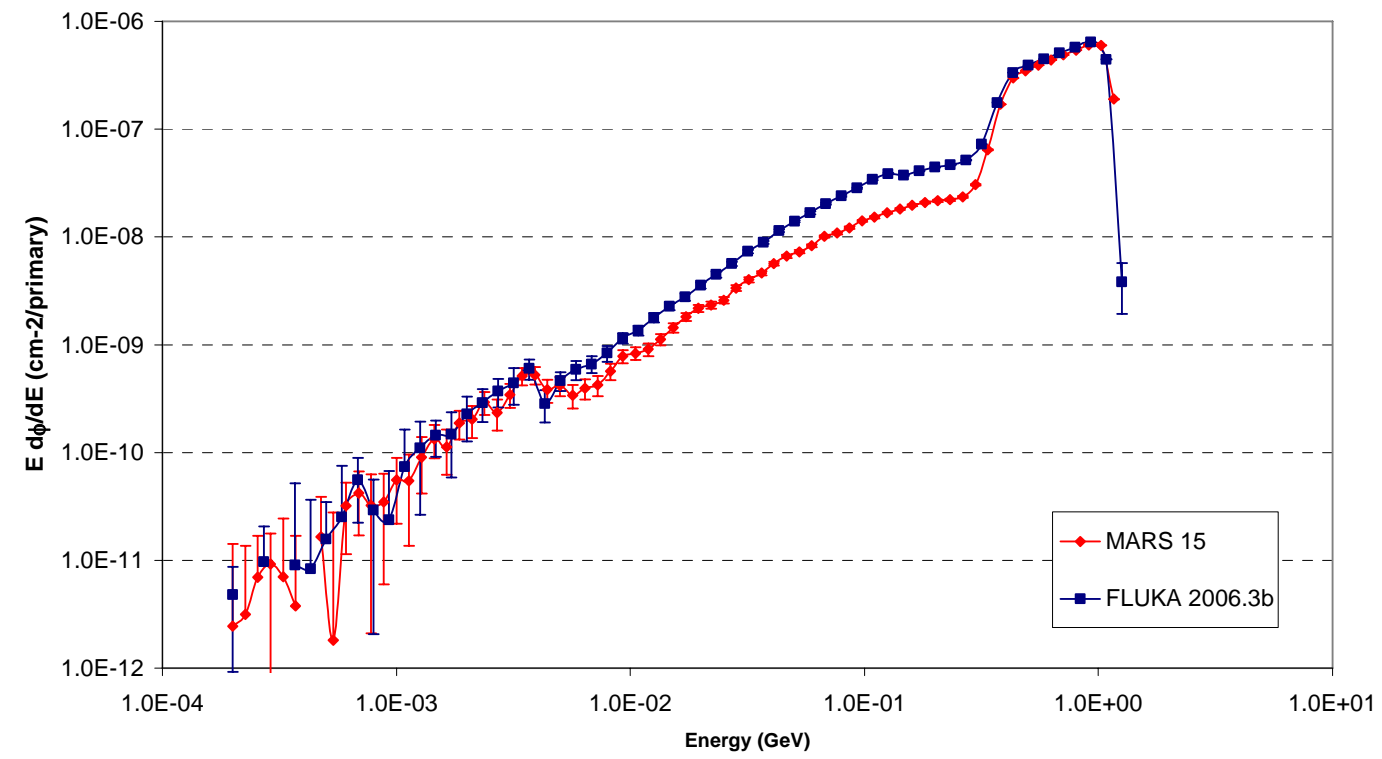

Figure 20: Energy spectra of the muons After the Iron Target 


\section{Conclusions}

The inter-comparison of the two principal codes - FLUKA and MARS - used for the LHC design and upgrades was performed for an identical model of the insertion region IR5. The model consists of the first part of the insertion region, with only one quadrupole magnet. Both codes use the event generator DPMJET III for simulating the proton-proton collisions. The results are normalized to an upgrade luminosity of $10^{35} \mathrm{~cm}^{-2} \mathrm{~s}^{-1}$.

The total heat loads calculated in the quadrupole agree for most of the components within $5 \%$, coinciding within $1 \%$ for the critical region (superconducting coils) and $23 \%$ for the yoke. The peaks in the inner cable are in a very good agreement within the statistical error $(<5 \%)$. The variations for power density along the superconducting cable closest to the beam pipe are well featured by the codes, with FLUKA predicting somewhat higher power densities at the non-IP end of the quadrupole. For particle spectra scored in the first superconducting coil, the comparative results are also encouraging with small differences that can be explained by differences in the particle production models and partly by the statistical errors.

To further investigate the muon production and transport models in FLUKA and MARS, a supplementary study has been made on a model of a $2-\mathrm{GeV}$ pion beam impacting a carbon target and an iron target separated by a drift. Here again the results are conclusive. FLUKA and MARS give similar longitudinal distributions of the muon fluence and similar shapes for the spectra even though the different hadron models of the two codes lead to some differences in the results.

\section{Acknowledgments}

The inter-comparison work was possible thanks to the fruitful collaboration between CERN (AT/MCS/MA and AB/ATB/EET) and Fermilab (Accelerator Physics Center). The visit at Fermilab for this collaboration was supported by the LARP program.

We would also like to thank the following persons for helpful information and discussions: Elena Wildner, Jean Pierre Koutchouk, Francesco Broggi. 


\section{References}

[1] 'Protecting LHC IP1/IP5 components against radiation resulting from colliding beam interactions’, N.V. Mokhov, I.L. Rakhno, J.S. Kerby, J.B. Strait, Fermilab, LHC report 633, April 2003.

[2] 'Mitigating radiation loads in Nb3Sn quadrupoles for the CERN Large Hadron Collider upgrades', N.V. Mokhov, I.L. Rakhno, PRSTAB, 9, 101001 (2006), see also Fermilab-FN0789-AD, July 2006.

[3] 'Study of the stability of the LHC low Beta inner triplet for a $\mathrm{Nb}_{3} \mathrm{Sn}$ design', G. Ambrosio, F. Broggi, L. Rossi, INFN report INFN/ TC-99/04, March 1999.

[4] 'Energy Deposition in Insertion regions IR1 and IR5', C.Hoa, F. Cerutti, LHC note to be published, November 2007.

[5] 'FLUKA: a multi-particle transport code', A. Fasso', A. Ferrari, J. Ranft, and P.R. Sala, CERN-2005-10 (2005), INFN/TC_05/11, SLAC-R-773.

[6] 'The physics models of FLUKA: status and recent developments',A. Fasso', A. Ferrari, S. Roesler, P.R. Sala, G. Battistoni, F. Cerutti, E. Gadioli, M.V. Garzelli, F. Ballarini, A. Ottolenghi, A. Empl and J. Ranft, Computing in High Energy and Nuclear Physics 2003 Conference (CHEP2003), La Jolla, CA, USA, March 24-28, 2003, (paper MOMT005), eConf C0303241 (2003), arXiv:hep-ph/0306267.

[7] 'The MARS Code System Users Guide', N.V. Mokhov, Fermilab-FN-628 (1995). http://www-ap.fnal.gov/MARS/.

[8] 'MARS15 Overview', N.V. Mokhov, S.I. Striganov, Fermilab-Conf-07/008-AD (2007); in Proc. of Hadronic Shower Simulation Workshop, Fermilab, September 2006, AIP Conf. Proc. 896, pp. 50-60 (2007). 'Physics Models in the MARS15 Code for Accelerator and Space Applications', N.V. Mokhov, K.K. Gudima, S.G. Mashnik et al, in Proc. of International Conference on Nuclear Data for Science and Technology, Santa Fe, NM, 2004, AIP Conf. Proc. 769, part 2, pp. 1618-1623; Fermilab-Conf-04/269-AD (2004).

[9] Hadronic Shower Code Inter-Comparison and Verification, N.V. Mokhov, S.I. Striganov, in Proc. Of Hadronic Shower Simulation Workshop, Fermilab, September 2006, AIP Conf. Proceedings 896, pp. 112-122 (2007).

[10] The FLUKA code: description and benchmarking G. Battistoni, F. Cerutti, A. Fass $\tilde{A}^{2}$, A. Ferrari, S. Muraro, J. Ranft, S. Roesler, and P. R. Sala, AIP Conf. Proc. 896, 31 (2007)

[11] FLAIR for FLUKA (FLUKA Advanced Interface), Vasilis Vlachoudis, March 2007, http://www.fluka.org/flair/index.html.

[12] DPMJET III event generator, Stefan Roesler, Ralph Engel and Johannes Ranft http://sroesler.web.cern.ch/sroesler/dpmjet3.html.

[13] ‘LHC Design report, Vol. I The LHC main ring’, CERN-2004-003, June 2004. 